\title{
The effect of drying methods on the chemical composition and in-vitro digestibility of some browse plants
}

${ }^{1}$ Akinyemi, B. T., ${ }^{1}$ Babatunde, M. O., ${ }^{1}$ Alli, J. A., ${ }^{1}$ Ogunsanya, O. A., ${ }^{1}$ Jolaosho, A. O., ${ }^{1}$ Arigbede, O. M., and ${ }^{2}$ Adelusi, O. O.

${ }^{I}$ Department of Pasture and Range Management, ${ }^{2}$ Department of Animal Nutrition, Federal University of Agriculture, Abeokuta, Ogun State.

Corresponding author: akinyemibt@gmail.com, akinyemibt@funaab.edu.ng

\begin{abstract}
$\overline{B r o w s e}$ plants are under-utilized in the dry season because of the insufficient knowledge about the drying process that best helps to prevent degradation of nutrients available to ruminant. The experiment was conducted to investigate the effects of drying methods (air drying, oven drying and sun drying) on the chemical composition, in vitro digestibility of leaves and leaves + twigs from (Albizia lebbeck, Albizia saman, Daniella oliveri, Enterolobium cyclocarpum, Gliricidia sepium, Leucaena leucocephala, Milletia griffoniana and Pterocarpus santalinoides). The leaves and twigs were dried under the three drying methods until constant weight and milled for in vitro digestibility. Gas production was recorded at $6,12,18,24,30,36,42$, and 48 hours of the incubation period with $8 \times 3 \times 2$ factorial design using Duncan Multiple Ranged test. When the browse species were compared, there were significant differences in the incubation hours while there were also significant $(P>0.05)$ differences for the plant parts in the incubation hours except at 3 hours. The value of short chain fatty acid (SCFA) $\mu \mathrm{mol}$, ranged from 0.22 to 0.57, metabolizable energy (ME) MJkgDM ranged from 2.72 to 3.25, while organic matter digestibility (OMD) \% ranged from 38.44 to 50.48 respectively. Albizia lebbeck was highest $(P<0.05)$ for $S C F A$, $M E, O M D$. It is concluded that drying methods had no effect on the forages considered. However, the leaves of these browse plants are highly digestible and their inherent nutrients are higher than the range recommended for the maintenance in ruminant production.
\end{abstract}

Keywords: In Vitro, Digestibility, Proximate composition, Browse plants, Dry methods, Plant species

\section{Introduction}

Nutrition is the most important consideration in ruminant production systems. Supply of feed in inadequate amount and quality is responsible to a large extent for the low livestock productivity in the tropics. Ruminant animals depend solely on plants for their nutrient requirement in general and energy in particular. As man competes with his livestock for edible grains, forage has been reported (Das and Ghosh, 2001) to contribute about $75 \%$ of the dietary needs of ruminants. There is a seasonal in variation availability of natural pasture, pasture tends to be more succulent, highly nutritious and more abundant in the rainy season (around May - November) as opposed to the dry season (November - April) where they become fibrous, scarce and devoid of most essential nutrients such as protein, energy, minerals and vitamins which are required for increased rumen microbial fermentation. The feeding value of any forage is a function of characteristics of the species, such as its availability, accessibility and nutrient availability. Browse plants species have high potentials as important feed resources for ruminants during the dry season, are quite palatable and less susceptible to a climate fluctuation. In ruminant production, the feeding value of forages depends on the balance between 
available nutrient and the quantity of the nutrient ingested by the animal (Matlebyane, 2005). The use of in vitro gas production technique therefore remains a better alternative for the determination of gas production kinetics and energy value of ruminant feedstuffs and is accurate for the prediction of the organic matter digestibility and escape of nutrients from the rumen (Ajayi and Babayemi, 2008). Hence, this present study assessed the nutritional characteristics based on their chemical composition and gas production of selected browse plants and plant parts.

\section{Materials and methods}

The experiment was carried out at the Laboratory of the Department of Pasture and Range Management, College of Animal Science and Livestock Production (COLANIM), Federal University of Agriculture, Abeokuta, Ogun State, Nigeria. The University is located in the derived savannah vegetation zone of South-western part of Nigeria (Latitude $7^{0}$ $13^{\prime} 49.46 \mathrm{~N}$, longitude $3^{0} 25^{\prime} 11.98^{\prime} \mathrm{E}$ ) with elevation of 415 feet and eye altitude of $7577 \mathrm{~m}$ above sea level (Google earth, 2017).

\section{Experimental design}

The experiment was $8 \times 3 \times 2$ factorial design which was replicated two times. It comprises of eight browse plants (Albizia lebbeck, Albizia saman, Daniella oliveri, Enterolobium cyclocarpum, Gliricidia sepium, Leucaena leucocephala, Milletia griffoniana and Pterocarpus santalinoides), three drying methods (air, sun and oven dry) and two plant parts (leaves, leaves + twigs).

\section{Sample collection}

The browse plants were collected during the early dry season of 2014 (OctoberDecember) in which the leaves, leaves and twigs of Albizia lebbeck, Albizia saman, Daniella oliveri, Enterolobium cyclocarpum, Gliricidia sepium, Leucaena leucocephala, Milletia griffoniana and Pterocarpus santalinoides were collected from the University farms.

\section{Sample preparation}

The browse species were harvested, sorted into leaves, leaves and twigs. The samples were subjected to three drying methods drying; Air drying at room temperature, Sun drying and oven drying at $65{ }^{\circ} \mathrm{C}$ until a constant weight was attained. Thereafter, the samples were hammer milled, labeled, packaged and stored in dry place for further laboratory analysis.

\section{In vitro gas production technique}

In vitro gas production, ME and OMD were determined according to Menke and Steingass (1988) and SCFA (Getachew et al., 1999).

\section{Statistical analysis}

All data obtained was subjected to one-way Analysis of variance (ANOVA) and the treatment means were separated using Duncan Multiple Ranged test.

\section{Results}

Table 1 shows the proximate composition of browse plants, drying methods and plant part. A significant difference was recorded in the dry matter (DM) of the browse plant which ranged from $81.89 \%$ in $A$. lebbeck to $66.85 \%$ in $P$. santalinoides. The crude protein (CP) recorded a significant difference which ranged from $17.28 \%$ in $A$. saman to $14.48 \%$ in E. cyclocarpum. Ether extract (EE) content was significant among the browse plants with $P$. santalinoides having the highest value of $9.25 \%$ and $A$. saman with the lowest value of 7.34. The ash content was significant among the browse plants with $D$. oliveri having the highest value of $12.11 \%$ and $A$. saman with the lowest value of $4.76 \%$. Drying methods were significant on the proximate composition of the browse plants except on the ADF and Cellulose content, with the 
ADF value ranging from $38.81 \%$ in oven dried to $37.27 \%$ in air dried and cellulose contents ranging from $24.68 \%$ in oven dried to $23.31 \%$ in sun dried. The plant part had significant effects on the proximate composition of browse plant except on the EE, NDF, ADF, Hemi-cellulose and cellulose content.

Table 1: Main effect of browse plant, plant parts and drying methods on chemical composition (\%)

\begin{tabular}{|c|c|c|c|c|c|c|c|c|c|}
\hline Browse & DM & $\mathbf{C P}$ & EE & ASH & NDF & ADF & ADL & HEM & CELL \\
\hline LL & $79.00^{b}$ & 15.40 & 8.11 & $8.44^{\mathrm{a}}$ & $58.22^{\mathrm{a}}$ & $44.14^{\mathrm{ab}}$ & $9.87^{\mathrm{ab}}$ & $14.08^{a}$ & $34.27^{\mathrm{a}}$ \\
\hline AS & $78.19^{\mathrm{a}}$ & $17.28^{\mathrm{a}}$ & $7.34^{b}$ & $4.76^{c}$ & 58.78 & $37.47^{\mathrm{ab}}$ & $14.57^{\mathrm{a}}$ & 21.31 & $22.91^{\mathrm{b}}$ \\
\hline $\mathrm{AL}$ & $81.89^{\mathrm{a}}$ & 15.64 & 7.56 & $7.56^{\mathrm{d}}$ & $53.28^{c}$ & $37.92^{\mathrm{c}}$ & $9.54^{\mathrm{bc}}$ & $15.36^{\mathrm{a}}$ & $28.38^{c}$ \\
\hline $\mathrm{DO}$ & $79.78^{b}$ & 14.75 & 7.89 & $12.11^{\mathrm{a}}$ & $56.92^{\mathrm{ab}}$ & $45.52^{\mathrm{a}}$ & $9.34^{\mathrm{c}}$ & $11.39^{\mathrm{b}}$ & $36.18^{\mathrm{a}}$ \\
\hline PS & $66.85^{\mathrm{c}}$ & $16.55^{\mathrm{c}}$ & $9.25^{\mathrm{a}}$ & $8.05^{\mathrm{a}}$ & 58.72 & $35.86^{\mathrm{b}}$ & $13.88^{\mathrm{ab}}$ & 22.86 & $21.98^{\mathrm{b}}$ \\
\hline $\mathrm{EC}$ & $75.69^{b}$ & $14.48^{\mathrm{d}}$ & $7.54^{\mathrm{b}}$ & $8.05^{\mathrm{a}}$ & 60.64 & $40.83^{\mathrm{a}}$ & $13.29^{b}$ & 19.81 & $27.08^{a}$ \\
\hline $\mathrm{MG}$ & $75.88^{\mathrm{b}}$ & $17.27^{\mathrm{b}}$ & $8.94^{\mathrm{a}}$ & $6.84^{\mathrm{b}}$ & 58.19 & $37.39^{\mathrm{ab}}$ & $13.54^{\mathrm{b}}$ & 20.81 & $23.85^{\mathrm{ab}}$ \\
\hline GS & $79.44^{\mathrm{b}}$ & 15.30 & 8.33 & $10.50^{\mathrm{b}}$ & $54.28^{b c}$ & $41.47^{b}$ & $10.19^{\mathrm{a}}$ & $12.97^{\mathrm{ab}}$ & $31.28^{\mathrm{b}}$ \\
\hline SEM & 0.204 & 0.001 & 0.328 & 0.094 & 1.285 & 1.334 & 0.319 & 1.414 & 1.357 \\
\hline \multicolumn{10}{|c|}{ Drying methods } \\
\hline $\mathrm{AD}$ & $74.29^{\mathrm{b}}$ & $19.44^{\mathrm{a}}$ & $9.00^{\mathrm{a}}$ & $7.18^{\mathrm{a}}$ & $57.77^{\mathrm{b}}$ & 37.27 & $13.05^{\mathrm{b}}$ & $24.48^{\mathrm{a}}$ & 23.85 \\
\hline OD & $75.23^{\mathrm{a}}$ & $16.68^{\mathrm{b}}$ & $8.30^{\mathrm{ab}}$ & $6.41^{\mathrm{b}}$ & $57.42^{\mathrm{b}}$ & 38.81 & $14.13^{\mathrm{a}}$ & $18.60^{\mathrm{b}}$ & 24.68 \\
\hline $\mathrm{SD}$ & $72.94^{\mathrm{c}}$ & $13.06^{\mathrm{c}}$ & $7.51^{\mathrm{b}}$ & $7.20^{\mathrm{a}}$ & $62.06^{\mathrm{a}}$ & 37.58 & $14.27^{\mathrm{a}}$ & $24.47^{\mathrm{a}}$ & 23.31 \\
\hline SEM & 0.177 & 0.001 & 0.284 & 0.082 & 1.113 & 1.155 & 0.276 & 1.224 & 1.175 \\
\hline \multicolumn{10}{|l|}{ Plant parts } \\
\hline Leaves & $75.55^{\mathrm{a}}$ & $17.59^{\mathrm{a}}$ & 8.23 & $7.10^{\mathrm{a}}$ & 59.17 & 37.81 & $13.37^{\mathrm{b}}$ & 21.36 & 24.20 \\
\hline Leaves+twigs & $72.76^{\mathrm{b}}$ & $15.20^{\mathrm{b}}$ & 8.31 & $6.76^{\mathrm{b}}$ & 59.00 & 37.97 & $14.27^{\mathrm{a}}$ & 21.03 & 23.70 \\
\hline SEM & 0.144 & 0.001 & 0.232 & 0.067 & 0.908 & 0.943 & 0.225 & 1.000 & 0.960 \\
\hline
\end{tabular}

Table 2 shows the main effect of browse plants, drying methods and plant parts on in vitro gas production which varied significantly from three to 48 hours of incubation. However, Albizia lebbeck produced high volume of gas from 3 hours of incubation until 48 hours of incubation. Gas production by Daniella oliveri was consistently high $(\mathrm{P}<0.05)$ throughout the incubation period from 6 to $48 \mathrm{hrs}$. Millettia griffoniana commenced gas production at six hour of incubation and ended up producing the lowest $(\mathrm{P}>0.05)$ volume of gas $(17.00 \mathrm{~mL} / 200 \mathrm{mg})$ at 48 hours of incubation. The plant parts varied consistently in the hour of incubation. At 18 hours, sun drying had highest $(\mathrm{P}<0.05)$ volume of gas production (16.66 $\mathrm{mL} / 200 \mathrm{mg}$ ) while air drying had the lowest volume of gas production (14.33 $\mathrm{mL} / 200 \mathrm{mg})$. There were significant $(\mathrm{P}<0.05)$ difference in the volume of gas produced in the leave compared to leaves + twigs. At 24 hours of incubation, the leaves had (20.88 mL/200mg) and ended up producing the highest $(\mathrm{P}<0.05)$ volume of gas $(29.10 \mathrm{~mL} / 200 \mathrm{mg})$ at 48 hours of incubation while leaves + twigs had lowest gas production $(23.48 \mathrm{~mL} / 200 \mathrm{mg})$ at 48 hours of incubation.

Table 3 shows the interactive effect of plant parts and browse plant species on in vitro gas production. The browse plants and plant parts on in vitro gas production varied significantly $(\mathrm{P}<0.05)$ from 3 hours until 48 hours of incubation (Table 3 ). At 3 hours, Leucaena leucocephala leaves + twigs had highest $(\mathrm{P}<0.05)$ volume of gas production 
The effect of drying methods on the chemical composition and in-vitro digestibility

Table 2: In vitro gas production of plant species, drying methods and plant parts

\begin{tabular}{lcccccc}
\hline $\begin{array}{l}\text { Browse } \\
\text { species }\end{array}$ & $\mathbf{6}$ & $\mathbf{1 2}$ & $\mathbf{1 8}$ & $\mathbf{2 4}$ & $\mathbf{3 6}$ & $\mathbf{4 8 ~ h o u r s}$ \\
& \multicolumn{7}{c}{} \\
\hline LL & $8.42^{\mathrm{a}}$ & $15.67^{\mathrm{a}}$ & $20.17^{\mathrm{ab}}$ & $22.50^{\mathrm{a}}$ & $26.67^{\mathrm{a}}$ & $30.00^{\mathrm{ab}}$ \\
AS & $3.92^{\mathrm{d}}$ & $7.58^{\mathrm{c}}$ & $10.42^{\mathrm{c}}$ & $12.25^{\mathrm{bc}}$ & $15.42^{\mathrm{c}}$ & $18.17^{\mathrm{c}}$ \\
AL & $7.92^{\mathrm{a}}$ & $16.67^{\mathrm{a}}$ & $22.92^{\mathrm{a}}$ & $26.42^{\mathrm{a}}$ & $31.17^{\mathrm{a}}$ & $35.33^{\mathrm{a}}$ \\
DO & $7.17^{\mathrm{ab}}$ & $14.75^{\mathrm{ab}}$ & $18.75^{\mathrm{b}}$ & $22.50^{\mathrm{a}}$ & $29.08^{\mathrm{a}}$ & $32.17^{\mathrm{ab}}$ \\
PS & $4.83^{\mathrm{cd}}$ & $9.00^{\mathrm{c}}$ & $13.08^{\mathrm{c}}$ & $16.83^{\mathrm{b}}$ & $22.58^{\mathrm{b}}$ & $27.33^{\mathrm{b}}$ \\
EC & $4.33^{\mathrm{d}}$ & $9.00^{\mathrm{c}}$ & $11.00^{\mathrm{c}}$ & $13.67^{\mathrm{bc}}$ & $16.00^{\mathrm{c}}$ & $18.33^{\mathrm{c}}$ \\
MG & $4.67^{\mathrm{d}}$ & $7.33^{\mathrm{c}}$ & $9.25^{\mathrm{c}}$ & $11.75^{\mathrm{c}}$ & $15.17^{\mathrm{c}}$ & $17.17^{\mathrm{c}}$ \\
GS & $6.08^{\mathrm{bc}}$ & $12.50^{\mathrm{b}}$ & $18.50^{\mathrm{b}}$ & $22.67^{\mathrm{a}}$ & $28.08^{\mathrm{ab}}$ & $31.83^{\mathrm{ab}}$ \\
SEM & 0.54 & 1.12 & 1.48 & 1.71 & 2.13 & 2.41 \\
Drying methods & & & & & & \\
AD & 5.47 & 10.66 & $14.13^{\mathrm{a}}$ & 17.38 & 21.44 & 25.22 \\
OD & 6.19 & 11.88 & $15.75^{\mathrm{ab}}$ & 18.56 & 22.91 & 25.56 \\
SD & 6.09 & 12.16 & $16.66^{\mathrm{a}}$ & 19.78 & 24.72 & 28.09 \\
SEM & 0.42 & 0.91 & 1.23 & 1.40 & 1.68 & 1.87 \\
Plant parts & & & & & & \\
Leaves & $6.60^{\mathrm{a}}$ & $13.23^{\mathrm{a}}$ & $17.54^{\mathrm{a}}$ & $20.88^{\mathrm{a}}$ & $25.77^{\mathrm{a}}$ & $29.10^{\mathrm{a}}$ \\
Leaves+twigs & $5.23^{\mathrm{b}}$ & $9.90^{\mathrm{b}}$ & $13.48^{\mathrm{b}}$ & $16.27^{\mathrm{b}}$ & $20.27^{\mathrm{b}}$ & $23.48^{\mathrm{b}}$ \\
SEM & 0.34 & 0.71 & 0.98 & 1.11 & 1.34 & 1.49 \\
\hline
\end{tabular}

${ }^{a, b, c}$ Means on the same column with different superscripts are significantly different $(\mathrm{P}<0.05)$

SEM = standard error of mean, $\mathrm{AD}=$ Air drying, $\mathrm{OD}=$ Oven drying, $\mathrm{SD}=$ Sun drying, $\mathrm{LL}=$ Leucaena leucocephala, $\mathrm{DO}=$ Daniella oliveri, $\mathrm{MG}=$ Milletia griffoniana, $\mathrm{AL}=$ Albizia lebbeck, $\mathrm{AS}=$ Albizia

Saman, PS = Pterocarpus Santalinoides, GS = Gliricidia sepium, EC = Enterolobium Cyclocarpum

(4.67 mL/200mg) while Albizia saman and Enterolobium cyclocarpum leaves + twigs had lowest gas production. However, Albizia lebbeck leaves produced high volume of gas from 3 hours of incubation until 48 hours of incubation. Albizia saman leaves + twigs commenced gas production at 3 hours of incubation and ended up producing the lowest $(\mathrm{P}>0.05)$ volume of gas $(14.67 \mathrm{~mL} / 200 \mathrm{mg})$ at 48 hours of incubation. Gas production by Gliricidia sepium leaves was consistently high $(\mathrm{P}<0.05)$ throughout the incubation period from 9 hours until 48 hours. 48 hours gas produced was similar $(\mathrm{P}<0.05)$ to 48 hours gas production of Albizia lebbeck leaves $(\mathrm{P}<0.05)$.

Table 4 shows the interactive effect of different drying methods and browse plant species on in vitro gas Production which varied consistently from 3 hour until 48 hours of incubation. At 3 hours, oven dried Leucaena leucocephala oven dry had highest $(\mathrm{P}<0.05)$ volume of gas production $(5.00 \mathrm{~mL} / 200 \mathrm{mg})$ while sun dried Pterocarpus santalinoides, oven dried Enterolobium cyclocarpum and oven dried Milletia griffoniana had lowest $(\mathrm{P}>0.05)$ volume of gas production. However, sun dried Albizia lebbeck produced high volume of gas $(46.50 \mathrm{~mL} / 200 \mathrm{mg})$ from 6 hours of incubation until 48 hours of incubation. Gas production from oven dried Gliricidia sepium was consistently high $(\mathrm{P}<0.05)$ throughout the incubation period from 6 hours until 48 hours of incubation. 48 hours gas volume produced (37.00 $\mathrm{mL} / 200 \mathrm{mg}$ ) from oven dried Gliricidia sepium was similar to 48 hours gas volume production of sun dried Albizia lebbeck. Oven dried Albizia saman commenced gas production at 3 hour of incubation $\mathrm{n}$ and ended up producing the lowest $(\mathrm{P}>0.05)$ volume of gas $(14.50 \mathrm{~mL} / 200 \mathrm{mg})$ at 48 hours of incubation. 
Akinyemi, Babatunde, Alli, Ogunsanya, Jolaosho, Arigbede and Adelusi

Table 3: Interaction effect of plant parts and browse plant species on in vitro gas production

\begin{tabular}{|c|c|c|c|c|c|c|c|}
\hline Browse & PP & 6 & 12 & 18 & 24 & 36 & 48 \\
\hline \multirow[t]{2}{*}{ LL } & $\mathrm{L}$ & $8.83^{a}$ & $17.00^{\mathrm{ab}}$ & $20.83^{b c}$ & $23.00^{\text {bcd }}$ & $26.17^{\text {bcdef }}$ & $29.00^{\text {bcde }}$ \\
\hline & $\mathrm{L}+\mathrm{T}$ & $8.00^{\mathrm{abc}}$ & $14.33^{b c}$ & $19.50^{\mathrm{bcd}}$ & $22.00^{\text {bcde }}$ & $27.17^{\text {bcde }}$ & $31.00^{\mathrm{bcd}}$ \\
\hline \multirow[t]{2}{*}{ AS } & $\mathrm{L}$ & $4.33^{\mathrm{de}}$ & $9.17^{\text {cdef }}$ & $12.67^{\text {efg }}$ & $14.83^{\mathrm{defg}}$ & $18.83^{\text {defghi }}$ & $21.67^{\text {def }}$ \\
\hline & $\mathrm{L}+\mathrm{T}$ & $3.50^{\mathrm{e}}$ & $6.00^{f}$ & $8.17^{f}$ & $9.67^{\mathrm{g}}$ & $12.00^{\mathrm{i}}$ & $14.67^{f}$ \\
\hline \multirow[t]{2}{*}{ AL } & $\mathrm{L}$ & $10.17^{\mathrm{a}}$ & $21.50^{\mathrm{a}}$ & $28.67^{\mathrm{a}}$ & $33.17^{\mathrm{a}}$ & $39.33^{\mathrm{a}}$ & $45.33^{a}$ \\
\hline & $\mathrm{L}+\mathrm{T}$ & $5.67^{\text {cde }}$ & $11.83^{\text {bcde }}$ & $17.17^{\text {bcde }}$ & $19.67^{\text {bcdef }}$ & $23.00^{\text {cdefgh }}$ & $25.33^{\text {cdef }}$ \\
\hline \multirow[t]{2}{*}{ DO } & $\mathrm{L}$ & $8.67^{\mathrm{ab}}$ & $16.17^{\mathrm{b}}$ & $20.17^{\mathrm{bc}}$ & $23.83^{\mathrm{bc}}$ & $30.67^{\mathrm{abc}}$ & $33.83^{b c}$ \\
\hline & $\mathrm{L}+\mathrm{T}$ & $5.67^{\text {cde }}$ & $13.33^{\mathrm{bcd}}$ & $17.33^{\text {bcde }}$ & $21.17^{\text {bcdef }}$ & $27.50^{\mathrm{bcd}}$ & $30.50^{\mathrm{bcd}}$ \\
\hline \multirow[t]{2}{*}{ PS } & $\mathrm{L}$ & $5.33^{\mathrm{de}}$ & $9.67^{\text {cdef }}$ & $14.33^{\text {cdef }}$ & $18.17^{\text {cdefg }}$ & $24.00^{\text {cdefg }}$ & $29.00^{\text {bcde }}$ \\
\hline & $\mathrm{L}+\mathrm{T}$ & $4.30^{\mathrm{de}}$ & $8.33^{\mathrm{def}}$ & $11.83^{\mathrm{ef}}$ & $15.50^{\text {cdef }}$ & $21.17^{\text {cdefg }}$ & $25.67^{\text {cdef }}$ \\
\hline \multirow[t]{2}{*}{$\mathrm{EC}$} & $\mathrm{L}$ & $4.50^{\mathrm{de}}$ & $8.67^{\mathrm{def}}$ & $10.83^{\mathrm{ef}}$ & $12.33^{\mathrm{fg}}$ & $15.67^{\mathrm{ghi}}$ & $18.17^{\mathrm{ef}}$ \\
\hline & $\mathrm{L}+\mathrm{T}$ & $4.17^{\mathrm{de}}$ & $9.33^{\text {cdef }}$ & $11.17^{\mathrm{ef}}$ & $14.00^{\text {efg }}$ & $16.33^{\text {fghi }}$ & $18.50^{\mathrm{ef}}$ \\
\hline \multirow[t]{2}{*}{ MG } & $\mathrm{L}$ & $4.67^{\mathrm{de}}$ & $8.17^{\mathrm{def}}$ & $10.00^{\mathrm{ef}}$ & $12.83^{\mathrm{fg}}$ & $16.83^{\text {efghi }}$ & $18.00^{\mathrm{ef}}$ \\
\hline & $\mathrm{L}+\mathrm{T}$ & $4.67^{\mathrm{de}}$ & $6.50^{\mathrm{ef}}$ & $8.50^{f}$ & $10.67^{\mathrm{g}}$ & $13.50^{\mathrm{hi}}$ & $16.33^{f}$ \\
\hline \multirow[t]{2}{*}{ GS } & $\mathrm{L}$ & $6.33^{\mathrm{bcd}}$ & $15.50^{\mathrm{b}}$ & $22.83^{\mathrm{ab}}$ & $27.83^{\mathrm{ab}}$ & $34.67^{\mathrm{ab}}$ & $37.83^{\mathrm{ab}}$ \\
\hline & $\mathrm{L}+\mathrm{T}$ & $5.83^{\text {cde }}$ & $9.50^{\text {cdef }}$ & $14.17^{\text {cdef }}$ & $17.50^{\text {cdefg }}$ & $21.50^{\text {cdef }}$ & $25.83^{\text {cdef }}$ \\
\hline SEM & & 0.25 & 0.53 & 0.72 & 0.81 & 0.98 & 1.08 \\
\hline
\end{tabular}

Table 4: Interaction effect of drying methods and browse plant species on in vitro gas production

\begin{tabular}{|c|c|c|c|c|c|c|c|}
\hline Browse & DM & 6 & 12 & 18 & 24 & 36 & 48 hours \\
\hline \multirow[t]{3}{*}{ LL } & $\mathrm{A}$ & $7.25^{\text {bcde }}$ & $15.50^{\text {bcde }}$ & $21.25^{\text {bcd }}$ & $23.50^{\mathrm{bcd}}$ & $28.00^{b c}$ & $31.00^{\text {bcde }}$ \\
\hline & $\mathrm{O}$ & $9.50^{\mathrm{ab}}$ & $14.75^{\text {bcdef }}$ & $19.50^{\text {bcdef }}$ & $22.75^{\text {bcde }}$ & $27.75^{b c}$ & $32.25^{\mathrm{bcd}}$ \\
\hline & S & $8.50^{\mathrm{abc}}$ & $16.75^{\mathrm{abc}}$ & $19.75^{\text {bcdef }}$ & $21.25^{\text {bcdef }}$ & $24.25^{\mathrm{bcd}}$ & $26.75^{\text {bcdef }}$ \\
\hline \multirow[t]{3}{*}{ AS } & A & $3.75^{\mathrm{fg}}$ & $6.75^{\mathrm{hi}}$ & $9.00^{\mathrm{gh}}$ & $11.25^{f}$ & $13.50^{\mathrm{d}}$ & $16.50^{\mathrm{ef}}$ \\
\hline & $\mathrm{O}$ & $4.75^{\text {defg }}$ & $8.00^{\text {fghi }}$ & $10.25^{\mathrm{fgh}}$ & $11.00^{\mathrm{f}}$ & $13.00^{\mathrm{d}}$ & $14.50^{\mathrm{f}}$ \\
\hline & $\mathrm{S}$ & $3.25^{\mathrm{g}}$ & $8.00^{\text {fghi }}$ & $12.00^{\text {defgh }}$ & $14.50^{\mathrm{def}}$ & $19.75^{\mathrm{bcd}}$ & $23.50^{\text {bcdef }}$ \\
\hline \multirow[t]{3}{*}{$\mathrm{AL}$} & A & $5.50^{\text {cdefg }}$ & $9.00^{\text {defghi }}$ & $11.75^{\mathrm{efgh}}$ & $15.25^{\mathrm{cdef}}$ & $19.50^{\mathrm{bcd}}$ & $24.75^{\text {bcdef }}$ \\
\hline & $\mathrm{O}$ & $6.75^{\text {bcdef }}$ & $18.50^{\mathrm{ab}}$ & $25.00^{\mathrm{ab}}$ & $27.50^{\mathrm{ab}}$ & $31.75^{\mathrm{ab}}$ & $34.75^{\mathrm{abc}}$ \\
\hline & S & $11.50^{\mathrm{a}}$ & $22.50^{\mathrm{a}}$ & $32.00^{\mathrm{a}}$ & $36.50^{\mathrm{a}}$ & $42.25^{\mathrm{a}}$ & $46.50^{\mathrm{a}}$ \\
\hline \multirow[t]{3}{*}{$\mathrm{DO}$} & A & 6.50 & $13.75^{\text {bcdefg }}$ & $18.50^{\text {bcdefg }}$ & $23.00^{\text {bcde }}$ & $29.00^{b c}$ & $33.00^{\mathrm{abcd}}$ \\
\hline & $\mathrm{O}$ & $7.00^{\text {bcdef }}$ & $14.50^{\text {bcdefg }}$ & $17.50^{\text {bcdefgh }}$ & $20.75^{\text {bcdef }}$ & $26.25^{\text {bcd }}$ & $27.75^{\text {bcde }}$ \\
\hline & S & $8.00^{\text {bcd }}$ & $16.00^{\mathrm{bcd}}$ & $20.25^{\text {bcde }}$ & $23.75^{\mathrm{bcd}}$ & $32.00^{\mathrm{ab}}$ & $35.75^{\mathrm{ab}}$ \\
\hline \multirow[t]{3}{*}{ PS } & A & $5.50^{\text {cdefg }}$ & $10.00^{\text {cdefghi }}$ & $13.50^{\text {cdefgh }}$ & $17.25^{\text {bcdef }}$ & $22.25^{\mathrm{bcd}}$ & $28.25^{\mathrm{bdf}}$ \\
\hline & $\mathrm{O}$ & $4.50^{\mathrm{efg}}$ & $8.00^{\text {fghi }}$ & $11.00^{\mathrm{efgh}}$ & $14.25^{\mathrm{def}}$ & $19.25^{\mathrm{bcd}}$ & $22.00^{\text {bcdef }}$ \\
\hline & S & $4.50^{\mathrm{efg}}$ & $9.00^{\text {efghi }}$ & $14.75^{\mathrm{cdefgh}}$ & $19.00^{\text {bcdef }}$ & $26.25^{\mathrm{bcd}}$ & $31.75^{\text {bcde }}$ \\
\hline \multirow[t]{3}{*}{$\mathrm{EC}$} & A & $3.75^{\mathrm{fg}}$ & 7.75 fghi & $9.75^{\mathrm{gh}}$ & $12.00^{\mathrm{ef}}$ & $13.75^{\mathrm{d}}$ & $16.50^{\mathrm{ef}}$ \\
\hline & $\mathrm{O}$ & $5.00^{\text {defg }}$ & $9.50^{\text {defghi }}$ & $12.00^{\mathrm{defgh}}$ & $14.75^{\mathrm{def}}$ & $17.00^{\mathrm{cd}}$ & $18.25^{\mathrm{def}}$ \\
\hline & S & $4.25^{\mathrm{efg}}$ & $9.75^{\text {defgh }}$ & $11.25^{\mathrm{efgh}}$ & $14.25^{\mathrm{def}}$ & $17.25^{\mathrm{cd}}$ & $20.25^{\mathrm{cdef}}$ \\
\hline \multirow[t]{3}{*}{ MG } & A & $5.25^{\text {cdefg }}$ & $8.75^{\text {efghi }}$ & $10.75^{\text {efgh }}$ & $13.50^{\text {def }}$ & $16.75^{\mathrm{cd}}$ & $19.75^{\text {cdef }}$ \\
\hline & $\mathrm{O}$ & $5.00^{\text {defg }}$ & $7.50^{\mathrm{gh}}$ & $9.00^{\mathrm{gh}}$ & $11.50^{f}$ & $15.75^{\mathrm{cd}}$ & $18.00^{\text {def }}$ \\
\hline & S & $3.75^{\mathrm{fg}}$ & $5.75^{\mathrm{i}}$ & $8.00^{\mathrm{h}}$ & $10.25^{f}$ & $13.00^{\mathrm{d}}$ & $13.75^{\mathrm{f}}$ \\
\hline \multirow[t]{3}{*}{ GS } & A & $6.25^{\text {bcdefg }}$ & $13.75^{\text {bcddefg }}$ & $18.50^{\text {bcdefg }}$ & $23.25^{\mathrm{bcd}}$ & $28.75^{b c}$ & $32.00^{\mathrm{bcd}}$ \\
\hline & $\mathrm{O}$ & $7.00^{\text {bcdef }}$ & $14.25^{\text {bcdefg }}$ & $21.75^{b c}$ & $26.00^{\mathrm{bc}}$ & $32.50^{\mathrm{ab}}$ & $37.00^{\mathrm{ab}}$ \\
\hline & S & $5.00^{\text {defg }}$ & $9.50^{\mathrm{defghi}}$ & $15.25^{\mathrm{cdefgh}}$ & $18.75^{\text {bcdef }}$ & $23.00^{\mathrm{bcd}}$ & $26.50^{\text {bcdef }}$ \\
\hline SEM & & 0.25 & 0.53 & 0.72 & 0.81 & 0.98 & 1.08 \\
\hline
\end{tabular}

$\overline{\mathrm{a}, \mathrm{b}, \mathrm{c}}$ Means on the same column with different superscripts are significantly different $(\mathrm{p}<0.05)$

$\mathrm{LL}=$ Leucaena leucocephala, $\mathrm{DO}=$ Daniella oliveri, $\mathrm{MG}=$ Milittia griffoniana

$\mathrm{AL}=$ Albizia lebbeck, $\mathrm{AS}=$ Albizia saman, $\quad \mathrm{PS}=$ Pterocarpus santalinoides, $\mathrm{GS}=$ Gliricidia sepium

$\mathbf{E C}=$ Enterolobium cyclocarpum $\mathbf{D M}=$ Drying methods $\mathbf{A}=$ Air $\mathbf{O}=$ Oven $\mathbf{S}=$ Sun

SEM $=$ Standard error of mean 
Table 5 shows the interactive effect of post incubation parameters of methane, IVDMD as influenced by browse species, drying methods and part of plants. Albizia lebbeck had the highest value for SCFA, OMD, ME and was significantly $(\mathrm{P}<0.05)$ different from other browse plants. There were significant $(\mathrm{P}>0.05)$ difference in short chain fatty acid, (SCFA), Organic matter digestibility (OMD) and Metabolizable energy (ME) of plant parts. There were no significant $(\mathrm{P}>0.05)$ difference in short chain fatty acid, (SCFA), Organic matter digestibility (OMD) and Metabolizable energy (ME) among the drying methods. The drying methods however had significant $(\mathrm{P}>0.05)$ influence on the short chain fatty acid, (SCFA), Organic matter digestibility (OMD) and Metabolizable energy (ME). The value of SCFA in $\mu \mathrm{mol}$, ranges from 0.33 in leaves + twigs to 0.44 in leaves and was significantly $(\mathrm{P}<0.05)$ different. The value of OMD in $\%$ ranges from 42.27 in leaves + twigs and 47.66 in leaves and was significantly $(\mathrm{P}<0.05)$ different. The value of metabolizable energy ( $\mathrm{ME}$ ) in $\mathrm{MJ} / \mathrm{Kg} \mathrm{DM}$ ranges from 2.88 in leaves + twigs and 3.05 in leaves and was significantly $(\mathrm{P}<0.05)$ different. The value obtained for methane gas in this present study showed significant $(\mathrm{P}<0.05)$ differences in methane gas production from browse plants. Albizia saman and Milletia griffoniana produced the least amount of methane $(9.17 \mathrm{ml})$ while the highest volume of $(29.83 \mathrm{ml})$ was recorded for Pterocarpus santalinoides. There were significant $(\mathrm{P}<0.05)$ difference in methane of the plant parts. The value obtained for the IVDMD were significantly $(\mathrm{P}<0.05)$ different among browse plants. IVDMD was higher for all browse plants with Pterocarpus santalinoides having the lowest $(32.83 \%)$ while the highest volume of $(42.17 \%)$ was recorded for Albizia lebbeck. There were also no significant differences in plant parts.

Table 5: Post incubation parameters of methane, IVDMD as influenced by browse species, drying methods and part of plants.

\begin{tabular}{llllll}
\hline Browse & SCFA $(\boldsymbol{\mu m o l})$ & ME (MJ/kg) & OMD (\%) & METHANE & IVDMD (\%) \\
\hline LL & $0.48^{\mathrm{a}}$ & $3.12^{\mathrm{a}}$ & $48.01^{\mathrm{a}}$ & $25.00^{\mathrm{b}}$ & $40.00^{\mathrm{cd}}$ \\
AS & $0.23^{\mathrm{bc}}$ & $2.72^{\mathrm{c}}$ & $42.09^{\mathrm{b}}$ & $9.17^{\mathrm{e}}$ & $39.33^{\mathrm{d}}$ \\
AL & $0.57^{\mathrm{a}}$ & $3.25^{\mathrm{a}}$ & $50.48^{\mathrm{a}}$ & $22.33^{\mathrm{c}}$ & $42.17^{\mathrm{a}}$ \\
DO & $0.48^{\mathrm{a}}$ & $3.11^{\mathrm{a}}$ & $49.86^{\mathrm{a}}$ & $24.67^{\mathrm{b}}$ & $40.83^{\mathrm{bc}}$ \\
PS & $0.34^{\mathrm{b}}$ & $2.90^{\mathrm{b}}$ & $41.69^{\mathrm{b}}$ & $29.83^{\mathrm{a}}$ & $32.83^{\mathrm{f}}$ \\
EC & $0.27^{\mathrm{bc}}$ & $2.77^{\mathrm{bc}}$ & $40.63^{\mathrm{b}}$ & $11.33^{\mathrm{d}}$ & $39.33^{\mathrm{d}}$ \\
MG & $0.22^{\mathrm{c}}$ & $2.73^{\mathrm{c}}$ & $38.44^{\mathrm{b}}$ & $9.17^{\mathrm{e}}$ & $41.67^{\mathrm{ab}}$ \\
GS & $0.48^{\mathrm{a}}$ & $3.12^{\mathrm{a}}$ & $48.49^{\mathrm{a}}$ & $11.33^{\mathrm{d}}$ & $36.00^{\mathrm{e}}$ \\
SEM & $\mathbf{0 . 0 4}$ & $\mathbf{0 . 0 6}$ & $\mathbf{2 . 1 8}$ & $\mathbf{1 . 7 1}$ & $\mathbf{0 . 7 0}$ \\
Drying Method & & & & & \\
AD & 0.36 & 2.93 & 45.13 & $18.94^{\mathrm{a}}$ & $39.50^{\mathrm{a}}$ \\
OD & 0.38 & 2.96 & 43.26 & $17.31^{\mathrm{b}}$ & $39.50^{\mathrm{a}}$ \\
SD & 0.41 & 3.01 & 46.49 & $17.31^{\mathrm{b}}$ & $38.06^{\mathrm{b}}$ \\
SEM & $\mathbf{0 . 0 3}$ & $\mathbf{0 . 0 5}$ & $\mathbf{0 . 0 3}$ & $\mathbf{1 . 6 0}$ & $\mathbf{0 . 6 1}$ \\
Plant parts & & & & & \\
L & $0.44^{\mathrm{a}}$ & $3.05^{\mathrm{a}}$ & $47.66^{\mathrm{a}}$ & $19.96^{\mathrm{a}}$ & 38.75 \\
L+T & $0.33^{\mathrm{b}}$ & $2.88^{\mathrm{b}}$ & $42.27^{\mathrm{b}}$ & $15.75^{\mathrm{b}}$ & 39.29 \\
SEM & $\mathbf{0 . 0 3}$ & $\mathbf{0 . 0 4}$ & $\mathbf{1 . 2 2}$ & $\mathbf{1 . 3 0}$ & $\mathbf{0 . 5 0}$ \\
\hline
\end{tabular}

SEM = Standard error of mean, $\mathbf{D M}=$ Drying methods, $\mathbf{A D}=$ Air drying $\mathbf{O D}=$ Oven drying, $\mathbf{S D}=$ Sun drying, $\mathbf{L}=$ Leaves

$\mathbf{L}+\mathbf{T}=$ Leaves + twigs, $\mathbf{L L}=$ Leucaena leucocephala, $\mathbf{D O}=$ Daniella oliveri, $\mathbf{M G}=$ Milletia griffoniana, $\mathbf{A L}=$ Albizia lebbeck, $\mathbf{A S}=$ Albizia saman, $\mathbf{P S}=$ Pterocarpus santalinoides, $\mathbf{G S}=$ Gliricidia sepium, $\mathbf{E C}=$ Enterolobium cyclocarpum,

IVDMD $=$ In vitro dry matter digestibility 


\section{Discussion}

Nutritional value of forages can be determined through the use of in vitro gas production techniques because the volume of gas produced by forages species reflects the end result of the fermentation of its substrate to volatile fatty acids (VFA), microbial biomass and neutralization of the VFA, thus demonstrating the nutritional value of such forages (Blummel and Becker, 1997). The value of 35.33 $\mathrm{ml} / 200 \mathrm{mgDM}$ obtained from this study was lower than that of $56.75 \mathrm{~mL} / 200 \mathrm{mgDM}$ of Arigbede, et al., (2008) for different parts of T. Africana. This shows that gas produced from this study may be attributed to many factors, it may be due to nature of the forage mixtures (Babayemi et al., 2004) and the rumen liquor used for incubation process. It is possible to obtain high gas production if animal from which the rumen fluid was obtained gets its nutrient requirement met, hence the level of carbohydrate of the forages might also determine the extent of degradation experienced as reported by Blummel and Becker, 1997. Rapidly fermentable carbohydrates yield higher propionate which is an indication of lower gas production and less digested forage materials unlike acetate which is a good indication of higher gas production and digestibility of the forage materials. Slower rates of drying in this study may promote loss of non-structural carbohydrates, loss of volatile organic substances, and protein degradation (Deinum and Maassen, 1994). Oven-drying are reported to induce physico-chemical changes mainly in the nitrogenous constituents of forage and this is likely to affect degradation (Abdalla, et al., 1988). Sun-air drying exposes the material to ultraviolent radiation, which reacts with forage constituent to reduce digestibility. This was particularly evident in the present study in which air drying and oven drying had the lowest digestibility value at the end of 48hours of incubation. This may be attributed to the period and hours spent during drying. It is, therefore, expected that slow-drying methods would result in higher proportions of NDF, ADF and ADL (Dzowela, et al., 1995) as recorded in the NDF, ADF and cellulose content of $D$. oliveri A. lebbeck, $L$. leucocephala air dried samples. The post incubation parameters (SCFA, ME, and OMD) are good indication of forage quality and digestibility because gas production is a reflection of the generation of short chain fatty acid (SCFA) and microbial mass (Getechew, et al., 1999). Gas volume had direct relationship with metabolizable energy of feed intake (Blummel and Becker, 1997) and growth rate of calves (Blummel and Orskov, 1993). The value 2.72 to $3.25 \mathrm{MJ} / \mathrm{KgDM}$ for metabolizable energy in this study was lower than that of (7.66 to $13.00 \mathrm{MJ} / \mathrm{KgDM}$ ) of Babayemi (2006) for foliages and fruits of Enterolobium cyclocarpum during dry season. This lower value of ME may be attributed to nutrient composition and season at which the browse plants were harvested and parts of the plant used. The value of ME (3.57 to 5.98) of Babayemi, et al. (2006). The value (38.44 to $50.48 \%$ ) of OMD of this study was lower than that of Babayemi (2006) for foliages and fruits of Enterolobium cyclocarpum (51.42 to 89.82 ). But the value (38.44 to $50.48 \%$ ) was similar to those of Babayemi and Bamikole 2006a) for Tephrosia candida and Guinea grass mixture and spent tea leaf (Babayemi et al., 2006). The value of SCFA in $\mu \mathrm{mol}$ of this study (0.22-0.57) was lower than $(0.69$ -1.68) of Babayemi (2006c) for foliages and fruits of enterolobium cyclocarpum but was similar to those of Tephrosia candida and guinea grass mixture of Babayemi and Bamikole, 2006) and spent tea leaf of Babayemi, et al. (2006). The lower value of SCFA, ME, and OMD of this study to that of 
Babayemi (2006) for foliages and fruit of Enterolobium cyclocarpum may be due to nutritional factor of browse species. Feedstuffs that are inherent with certain anti-nutritive factor had reported to be low in metabolizable energy and organic matter digestibility (Aregheore and Abdulrazak, 2005). Methane production is energy loss to ruminants and also contributes to global warming (Babayemi and Bamikole, 2006). The least amounts of methane gas were observed in both Albizia saman and Millettia griffoniana and it can be attributed to their rich condensed tannins. Hess, et al. (2006) reported that inhibition of methanogenesis by tannin was probably the result of a suppression of fibre degradation. The methane gas obtained for plant parts under this study were generally higher. The higher IVDMD observed may be due to the low level of tannin in the browse plants which suggest that it could be a valuable protein supplement in ruminant diets (Aganga and Mosase, 2001).

\section{Conclusion}

From this study, it can be concluded that the chemical composition of the plant parts, leaves recorded higher proximate content while the leaves with twigs were observed to have higher fibre contents, therefore it can be said that the leaves are of more nutritive value and will be more acceptable to the animals and preferable for on-farm feed processing and conservation. Since oven drying was recorded to have average effects on the chemical composition of the selected browse plants, it is concluded that oven-drying can be used in place of freezedrying (which is regarded as the golden drying method) for those laboratories that cannot afford the capital outlay required to purchase freeze-drying equipment. The high In vitro dry matter digestibility observed especially in Albizia lebbeck indicates that the plant have the potential of being used as protein supplement by ruminants fed low quality roughages especially during the dry season.

\section{References}

Abdalla, H. O., Fox, D. G. and Van Soest, P. J. 1988. An evaluation of methods for preserving fresh forage samples before protein fraction determination. Journal of Animal Science, 66, 2646-2649.

Aganga, A. A. and Mosase, K. W. 2001. Tannin content, nutritive value and dry matter digestibility of Lonchocarpus capassa, Zizyphus mucronata, Sclerocarya birrea, Kirkia acuminate and Rhus lancea seeds. Animal Feed Science and Technology. 91 (12): 107-113.

Ajayi, F. T. and Babayemi, O. J. 2008. Comparative in vitro evaluation of mixtures of panicum maximum cv Ntchisi with Stylo (Stylosanthes guianensis), Lablab (Lablab purpureus), Centro (Centrosema pubescen) and $\mathrm{H}$ istrix (Aeschynomene histrix), Livestock Research for Rural Development. $20(6)$.

Aregheore, E. M. and Abdulrazak, S. A. 2005. Estimation of organic matter digestibility and metabolizable energy content of agro-industrial wastes using in vitro gas production. Nigerian Journal of Animal Production 32(1):79-87.

Arigbede, O. M., Anele, U. Y., Oduguwa, B. O., Jolaosho, O. A., Olanite, J. A., Onifade, O. S., 2008. Replacement value of soybean haulms meal in the diets of West African Dwarf. Arch. Zootec., 57 (219): 369-372.

Babayemi, O. J. 2006. Anti-nutritional 
Factors, Nutritive value and In vitro Gas Production of foliage and Fruit of Enterolobium cyclocarpum. World Journal of Zoology, 1(2), 113-117.

Babayemi, O. J. and Bamikole M. A. 2006. Supplementary values of Tephrosia bracteolate, Tephrosia candida, Leucaena leucocephala and Gliricidia sepium hay for West African Dwarf goats kept on range. Journal of Central European Agriculture 7 (2): 323.

Babayemi, O. J., Demeyer, D. and Fievez, V. 2004. Nutritional value of qualitative assessment of secondary compound in seeds of eight tropical browse, shrub and pulse legumes. Comm. Applied Biological Science, Ghent University, 69/1: 103-110.

Blummel, M. and Becker, K. 1997. The degradability characteristics of fifty four roughages and roughage neutral detergent fiber as described in in vitro gas production and their relationship to voluntary feed intake. Brit, J. nutri. 77:757-768.
Das, A. and Ghosh, S. K. 2001. Nutritive value of jack fruit tree leaves for goats. Indian Journal of Animal Nutrition 18: 185-186.

Deinum, B. and Maassen. A. 1994. "Effects of drying temperature on chemical composition and in vitro digestibility of forages." Animal Feed Science and Technology 46: 75-86.

Dzowela, B. H., Hove, L. and Mafongoya P. L. 1995. Effect of drying method on chemical composition and in vitro digestibility of multi-purpose tree and shrub fodders.Tropical Grasslands. Volume 29, 263-269.

Hess, H. D., Tiemann, T. T., Noto, F., Carulla., J. E. and Kreuzer, M. 2006. Strategic use of tannins as means to limit methane emission from ruminant livestock. International Congress Series, 1293:164-167.

Received: $3^{\text {rd }}$ November, 2019 Accepted: $17^{\text {th }}$ February, 2020 